\title{
CORRELATION BETWEEN INFLAMMATORY SIGNALS AND TUMOR ASSOCIATED MACROPHAGES IN ORAL SQUAMOUS CELL CARCINOMA
}

\author{
Sarah Adolf Barsoom*, Houry Moustafa Baghdadi**, Hala A. El-kammar ${ }^{* * *}$ and Nermeen S Afifi****
}

\begin{abstract}
Background: Squamous cell carcinoma grows in a complex and dynamic stroma. This microenvironment provides all the necessary stimuli for tumor viability, growth and invasiveness. COX-2 is upregulated during both inflammation and cancer. CD163, a marker of M2 macrophages (Tumor associated macrophages- TAMs), has been studied in several aggressive tumors. The increased expression of CD163 was associated with a poor overall survival in various cancers.
\end{abstract}

Aims of the Study: The present study aimed to correlate the inflammatory signals presented by COX-2 immunoexpresion with tumor associated macrophages expressing CD163 in different grades of OSCC.

Materials and methods: Immunohistochemical expression of COX-2 and CD163 was evaluated in 30 cases of different grades of OSCC.

Results: The mean difference between the well-differentiated level and the moderately differentiated level of COX-2 and CD 163 was statistically insignificant, while that between the well- and poorly differentiated and between the moderately and the poorly differentiated were highly statistically significant.

Conclusions: inflammatory signals presented by COX-2 immunoexpression and TAM (CD163+ cells) were elevated with increased tumor grade.

KEY WORDS: COX-2, Tumor associated macrophages, CD 163, Squamous cell carcinoma

\section{INTRODUCTION}

Oral squamous cell carcinoma (OSCC) is one of the most prevalent cancers. Its five year survival rate has not improved greatly ${ }^{[1]}$. Squamous cell carcinoma grows in a complex and dynamic stroma. This microenvironment provides all the necessary stimuli for tumor viability, growth and invasiveness $^{[2]}$.

\footnotetext{
* Teaching Assistant of Oral Pathology, Faculty of Oral and Dental Medicine, Future University in Egypt

** Professor and Head of Oral Pathology Department, Faculty of Dentistry, Ain Shams University

*** Lecturer of Oral Pathology, Faculty of Oral and Dental Medicine, Future University in Egypt.

**** Lecturer of Oral Pathology, Faculty of Dentistry, Ain Shams University
} 
Virchow, in 1867, was the first to describe the role of inflammation in cancer progression ${ }^{[2]}$. It is well admitted that the link between chronic inflammation and cancer involves cytokines and mediators of inflammatory pathways, which act during the different steps of tumorigenesis. The cyclo-oxygenases (COXs) are a family of enzymes involved in inflammation ${ }^{[3,4]}$.

COX-2 is upregulated during both inflammation and cancer, which was described to modulate cell proliferation and apoptosis, mainly in solid tumors. It catalyzes the rate limiting steps of prostaglandin E2 (PGE2) biosynthesis from arachidonic acid which is released from the plasma membrane during inflammatory processes ${ }^{[3,5]}$.

There is a correlation between tumor growth and prostaglandin biosynthesis. Tumorigenesis is characterized by a disequilibrium between cell proliferation and cell death. PGE2 is able to inhibit apoptosis through the upregulation of Bcl-2. Added to that, PGE2 can upregulate the level of vascular endothelial growth factor (VEGF) and lead to activation of mitogen-activated protein kinases ${ }^{[6]}$.

Altogether, these data suggest that PGE2 and COX-2 play an important role in tumor progression by enhancing cell proliferation, cell survival and tumor invasion ${ }^{[?]}$.

Within the cancer milieu, tumor infiltrating immune cells play an important role in anticancer immunity. Macrophages are involved in various aspects of host defense mechanisms and pathophysiological conditions, such as chronic inflammatory diseases and cancer ${ }^{[8]}$. The functions of tumor-associated macrophages (TAMs) within the tumor site are various. Previously, TAMs were believed to exhibit antitumor activity by exerting direct cytotoxic effects on tumor cells and also by phagocytosing apoptotic cells and waste products ${ }^{[2]}$.

However, many recent studies have indicated the protumoral functions of TAMs ${ }^{[10-13]}$. Now, it became clear that monocytes can differentiate into "friendly" M1 macrophages, which initiate tumor rejection by releasing tumoricidal products, such as reactive oxygen intermediates (ROI), which kill tumor cells or "foe" M2 (CD163+) macrophages, which stimulate tumor growth, metastasis and angiogenesis $^{[14]}$.

CD163, a marker of M2 macrophages, has been studied in several aggressive tumors, and the increased expression of CD163 was associated with a poor overall survival in various cancers.

The present study aimed to correlate the inflammatory signals presented by COX-2 immunoexpresion with tumor associated macrophages expressing CD163 in different grades of OSCC.

\section{MATERIALS AND METHODS}

Immunohistochemical techniques using monoclonal antibodies to the specific isoenzymes COX-2 and CD163 were employed in this study. Paraffin blocks were cut at four micrometer thick sections were mounted on positively charged glass slides. Sections were deparaffinized with xylene and rehydrated in graded alcohol. Sections were immersed in citrate buffer and treated in a microwave before the staining procedures. For immunostaining, a universal kit (Lab Vision, USA) was used. Peroxidase-antiperoxidase method of immunostaining using the biotin-streptavidin system was carried out. $3 \%$ hydrogen peroxide was applied to the section to block endogenous peroxidase activity. The sections were immunolabelled using the primary antibodies COX-2 and CD163 (Lab Vision, USA) and then incubated overnight at room temperature. After rinsing with phosphate buffer saline (PBS), sections were covered by the link antibody, followed by streptavidin labeling antibody. After rinsing with PBS, diaminobenzidine chromogen was applied to the sections followed by the counterstain. Sections were dehydrated in graded alcohol, cleared in xylene and mounted.

All the steps for immunohistochemical quantitative evaluation were carried out on photomicrographs captured at a magnification of X20 using image analysis software (Image J, 1.41a, NIH, USA). 
Statistical package, IBM SPSS Statistics $22.0^{\mathrm{TM}}$, was utilized for the analysis of the area fraction results for both COX-2 and CD163 antibodies. One-way ANOVA followed by Tukey post-hoc tests were used for the comparison between the means of the different grades of both COX-2 and CD163 antibody. Also, the correlation between the grades of the COX-2 antibody and its corresponding CD163 antibodies grades were tested and coefficients of correlation calculated. All statistical analyses were calculated at a 0.05 significance level (p-value of 0.05 )

\section{RESULTS}

\section{Immunohistochemical Results}

\section{COX -2}

Immunohistochemical staining with monoclonal antibody against COX-2 was assessed. The positive reaction was brown in color, granular in nature and both nuclear and cytoplasmic in localization. All the 30 cases $(100 \%)$ of various grades of OSCC examined in this study showed COX-2 immunopositivity (figs. $1: A, B, C$ ).

In well and moderately differentiated OSCC, some of the malignant epithelial cells forming cell nests and keratin pearls revealed positive COX-2 immunostaining. The reaction was cytoplasmic, yet it should be noted that some of the immunopositive cells exhibited both cytoplasmic and nuclear immunoreactivity. There was focal immunopositivity around the cell nest for COX-2 in the connective tissue cells. The immunopositive stromal cells revealed cytoplasmic and nuclear immunoreactivity (fig.1:A,B).

In poorly differentiated OSCC, the individual malignant epithelial cell exhibited mainly cytoplasmic immunoreactivity and only a few cells showed cytoplasmic and nuclear reactions. There was focal immunopositivity for COX-2 in the connective tissue cells. The immunopositive stromal cells revealed cytoplasmic and nuclear reactions (fig. 1:C).

\section{CD163}

Immunohistochemical staining with monoclonal antibody against CD163 was assessed. The positive reaction was brown in color, granular in nature and both nuclear and cytoplasmic in localization. All the 30 cases $(100 \%)$ of various grades of OSCC, examined in this study showed CD163 immunopositivity (figs. 1:D,E,F).

In well and moderately differentiated OSCC, the distribution of the immunopositive cells was mainly in the connective tissue stroma. The immunopositive stromal cells exhibited granular cytoplasmic and nuclear immunopositivity. There were few malignant epithelial cells exhibiting cytoplasmic CD163 immunoreactivity (figs. 1:D,E).

In the poorly differentiated OSCC, the immunoreactivity was more diffuse than in the well and moderately differentiated OSCC. The expression was mostly noted in the stromal cells. The positive reaction was granular in nature, cytoplasmic and nuclear in localization (fig.1:F).

\section{Statistical results}

\section{COX-2 and CD 163 Results}

The mean and standard deviation of the three grades (well-, moderate - and poorly differentiated OSCC) are listed in table 1 and fig. 2.

One-way ANOVA test was used for the simultaneous comparison between the expression of both COX-2 and CD163 in the three grades of OSCC, and the differences between the mean of the three grades was found to be highly statistically significant at a p-value of less than 0.001 (table 2). Meanwhile, Tukey post-hoc test was used for the multiple comparisons between each pair of the grade levels of COX-2 and CD163. The mean difference between the well-differentiated level and the moderately differentiated level of COX-2 was statistically insignificant at a p-value of 0.289 and p-value of 0.601 for CD163, while, that between the well- and poorly differentiated and between the moderately- and the poorly differentiated were 


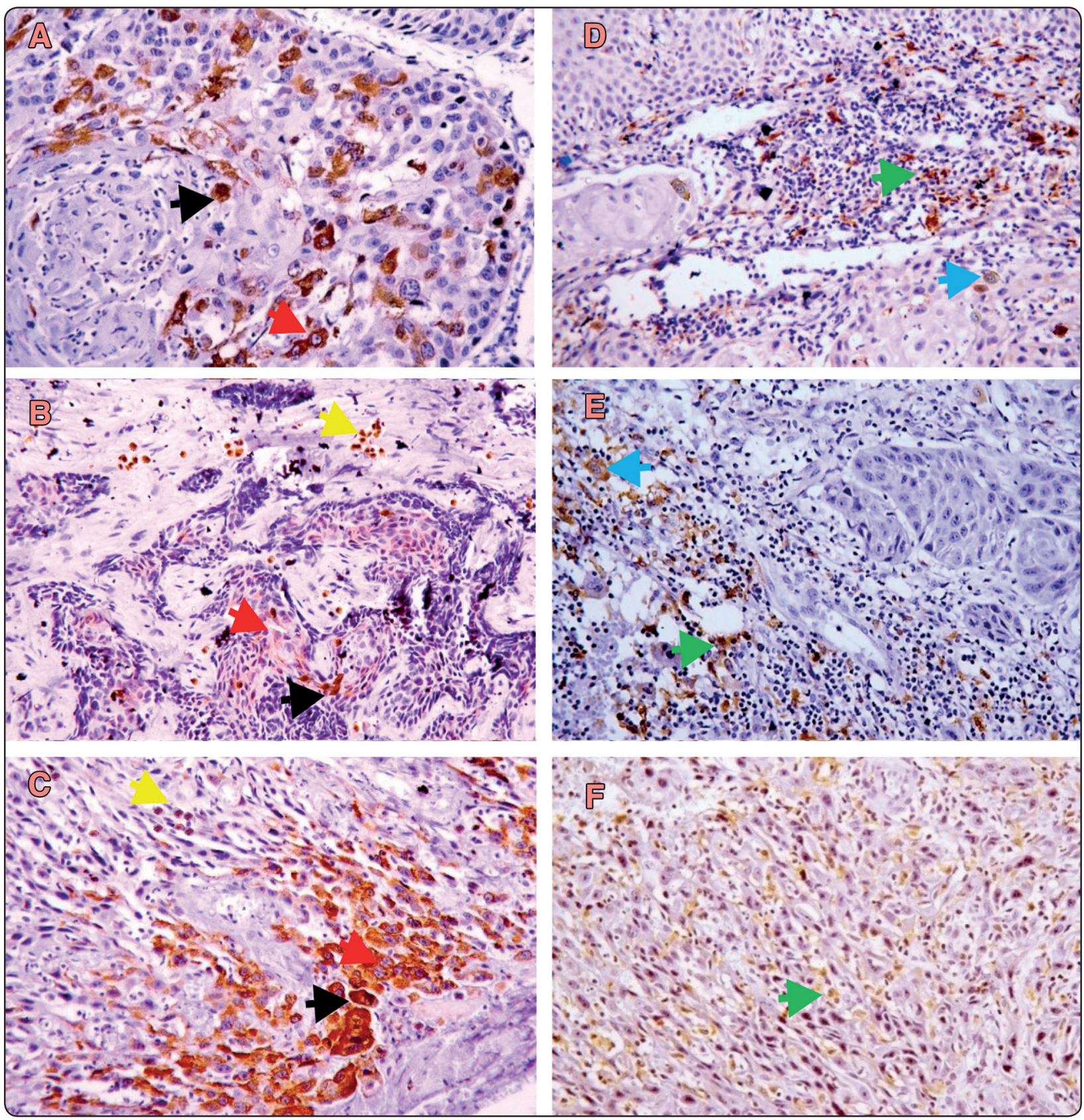

Fig. (1) Photomicrographs of COX-2 in well, moderate and poorly differentiated OSCC (A,B,C) and CD163 in well, moderate and poorly differentiated OSCC (D,E,F). The black arrows show nuclear and cytoplasmic COX-2 immunopositivity in malignant epithelial cells. The red arrows show cytoplasmic COX-2 immunopositivity in malignant epithelial cells. The yellow arrows show nuclear and cytoplasmic COX-2 immunopositivity in some of the stromal cells. The green arrows show nuclear and cytoplasmic CD163 immunopositivity in the stromal cells while the blue arrows show CD163 immunopositivity in few individual malignant epithelial cells. (Orig. Mag. X40) 
highly statistically significant at p-values less than 0.001 (table 3).

TABLE (1) Mean and standard deviation of the area fraction for different grades of OSCC using COX-2 and CD163.

\begin{tabular}{|l|c|c|c|}
\hline COX-2 & Mean & N & $\begin{array}{c}\text { Standard } \\
\text { deviation }\end{array}$ \\
\hline Well differentiated OSCC & 0.3529 & 10 & 0.3172 \\
\hline $\begin{array}{l}\text { Moderately differentiated } \\
\text { OSCC }\end{array}$ & 1.9237 & 10 & 0.8971 \\
\hline Poorly differentiated OSCC & 6.9704 & 10 & 3.8373 \\
\hline CD 163 & Mean & N & $\begin{array}{c}\text { Standard } \\
\text { deviation }\end{array}$ \\
\hline Well differentiated OSCC & 1.5778 & 10 & 0.6613 \\
\hline $\begin{array}{l}\text { Moderately differentiated } \\
\text { OSCC }\end{array}$ & 2.3722 & 10 & 1.0871 \\
\hline Poorly differentiated OSCC & 7.4067 & 10 & 2.9013 \\
\hline
\end{tabular}

TABLE (2) One-way ANOVA results of the area fraction for the different grades of OSCC using COX-2 and CD 163

\begin{tabular}{|l|c|c|c|}
\hline \multicolumn{1}{|c|}{ COX-2 } & $\begin{array}{c}\text { Sum of } \\
\text { squares }\end{array}$ & P-value & Significance \\
\hline Between Groups & 239.093 & $<0.001$ & S \\
\hline Within Groups & 140.672 & & \\
\hline Total & 379.765 & & \\
\hline \multicolumn{1}{|c|}{ CD 163 } & $\begin{array}{c}\text { Sum of } \\
\text { squares }\end{array}$ & P-value & Significance \\
\hline Between Groups & 199.844 & $<0.001$ & S \\
\hline Within Groups & 90.330 & & \\
\hline Total & 290.175 & & \multicolumn{1}{|c|}{} \\
\hline
\end{tabular}

\section{Correlation between COX-2 and CD163}

Correlation between the different grades of COX-2 and the corresponding CD163 grades were investigated (table 4). The well-differentiated grades for COX-2 and the corresponding CD163 grades were highly correlated with $r=0.954$. Also, a high correlation between the moderately-differentiated grades of both COX-2 and CD163 was found with $r=0.994$. Again, the poorly differentiated grades of COX-2 and CD163 were highly correlated, with $r=$ 0.967 (fig. 3). 
TABLE (4) Correlation between COX-2 and CD163 in different grades of OSCC

\begin{tabular}{|l|c|c|c|}
\hline $\begin{array}{c}\text { Compared } \\
\text { pair level }\end{array}$ & $\begin{array}{c}\text { Coefficient of } \\
\text { correlation, } \boldsymbol{r}\end{array}$ & P-value & Significance \\
\hline $\begin{array}{l}\text { Well } \\
\text { differentiated } \\
\text { OSCC }\end{array}$ & 0.954 & $<0.001$ & $\mathrm{~S}$ \\
\hline $\begin{array}{l}\text { Moderately } \\
\text { differentiated } \\
\text { OSCC }\end{array}$ & 0.994 & $<0.001$ & $\mathrm{~S}$ \\
\hline $\begin{array}{l}\text { Poorly } \\
\text { differentiated } \\
\text { OSCC }\end{array}$ & 0.967 & $<0.001$ & $\mathrm{~S}$ \\
\hline
\end{tabular}

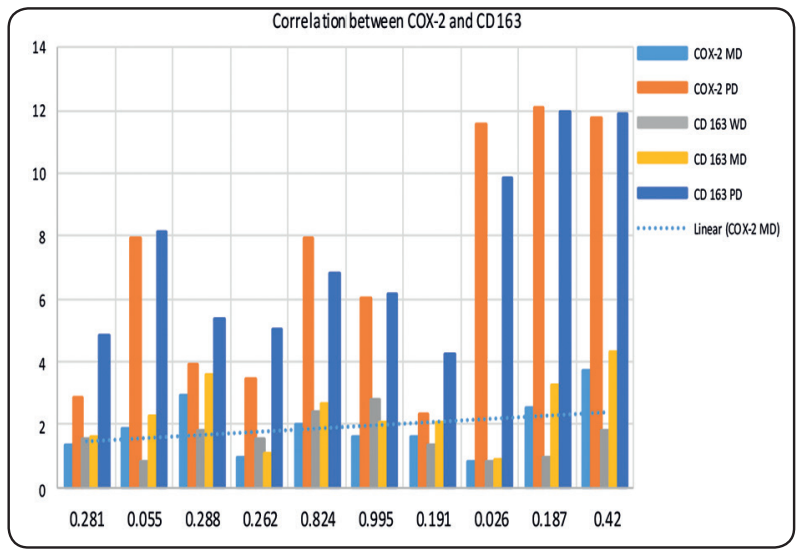

Fig. (3) COX-2 vs. CD 163 in different grades of OSCC

\section{DISCUSSION}

Squamous cell carcinoma (SCC) in the head and neck region occurs primarily in the oral cavity and oropharynx and is generally regarded as a disease of the elderly ${ }^{[1]}$. OSCC represents $95 \%$ of all forms of head and neck cancer, and during the past decade its incidence has increased ${ }^{[15]}$.

A connection between inflammation and the development of cancer has been studied heavily over the years. Epidemiological data indicate a link between inflammation and a predisposition for cancer development ${ }^{[16]}$.
Nevertheless, there is still debate about the impact of inflammation on cancer. Some researchers stated that inflammation correlates negatively with cancer stage and prognosis $[2,17,18]$. The role of inflammation has first come to attention when the use of nonsteroidal anti-inflammatory drugs (NSAIDs) helped in the prevention of spontaneous tumor formation in people with familial adenomatous polyposis ${ }^{[19]}$.

COX-2 is an inflammatory marker. The relationship between COX-2 expression and tumor differentiation in SCCs is still controversial. In the lungs, esophagus and larynx, it was reported that COX-2 expression was elevated in well-differentiated carcinomas more than in poorly differentiated ones ${ }^{[20]}$. Other studies showed that there was no correlation between the expression of COX-2 and the grade of malignancy. Itoh S. et al.(2003) ${ }^{[21]}$, Pandey M. et al. (2008) $)^{[22]}$ and Goulart J. et al. (2009) $)^{[23]}$ documented that no significant difference was observed in COX-2 expression between different grades of OSCC.

On the other hand, Urade M.(2008) $)^{[24]}$ reported that more undifferentiated SCCs (histological grade III) had a significantly higher COX-2 expression than grade I or II cases. Another study, as well, showed that a differentiation-inducing agent caused promotion of cell differentiation and suppression of cell growth and COX-2 expression ${ }^{[24]}$.

There are several contradicting results regarding COX-2 expression and its correlation to SCC grade, that is why this study attempted to re-evaluate this correlation.

Recently, tumor microenvironment (TME) has been the focus of intense research. TME compromises several connective tissue components, among which are the recently described CAFs and TAMs. Zaynagetdinov R. et al. (2011) ${ }^{[25]}$, Zhang J. et al. (2015) $)^{[26]}$, Guo Q. et al.(2016) ${ }^{[10]}$ and Yang L. and Zhang Y. (2017) ${ }^{[14]}$ suggested that TAMs are positively correlated with the histological grade of malignancy and the number of cancer stem cells 
(CSCs) found. TAMs are commonly identified by expression of CD163, CD204 or CD206 $6^{[14,27]}$.

In the present study, COX-2 and CD163 expressions were evaluated and correlated with different tumor grades of OSCC. This study also correlated the expression of COX-2 and CD163 to evaluate their association with each other in the different tumor grades of OSCC.

COX-2 expression in this study was similar to the above-mentioned studies where the immunopositive malignant epithelial cells exhibited a cytoplasmic reaction. However, it was noted - in this study- that there was nuclear expression of COX-2 in some of the malignant epithelial cells and that the number of cells with nuclear expression decreased in tumors with advanced grade. This may be an important point to consider as not just the number of positive cells but also the localization of COX-2 may be a prognostic factor.

Nagatsuka M. et al. (2012) ${ }^{[28]}$ noted that the expression of COX-2 was intense and diffuse in poorly differentiated OSCC, while well and moderately differentiated OSCC showed local staining around nests. Chiu H. et al. (2017) ${ }^{[29]}$ noted that COX-2 expression in stromal cells, including macrophages, some neutrophils, fibroblasts and vascular endothelial cells, was stronger at the edge of the lesion and also noted that COX-2 was expressed homogeneously in the cytoplasm of all OSCC grades, as in the study by Shirahama T. and Sakaakura C. (2001) $)^{[30]}$.

Parafenova H. et al.(2001) ${ }^{[31]}$, revealed in their study that stimulation of cells from quiescent state coincided with the trafficking of COX-2 from the nucleus to the cytoplasm which may indicate an impact of COX-2 localization on cellular gene expression.

The results of this study showed that the expression of COX-2 was significantly higher in the poorly differentiated OSCC than the well and moderately differentiated ones. This result was consistent with those of Masferrer J. et al. (2000) ${ }^{[32]}$,Renkonen J. et al. (2002) ${ }^{[33]}$, Shibata M. et al. $(2005)^{[34]}$,Urade M. (2008) $)^{[24]}$, Nagatsuka M. et al. (2012) ${ }^{[28]}$ and Chiu M. et al. (2017) ${ }^{[29]}$, who proved that increased COX-2 expression correlated to the histological grades of the tumor.

A similar correlation between tumor grade and COX-2 expression was observed in this study. Nevertheless, it should be noted that the increased COX-2 expression in the moderately differentiated OSCC over the well differentiated OSCC was statistically insignificant in this study.

The variation in expression may be due to the fact that the expression of COX-2 during oral carcinogenesis may depend on the developmental stage of the tumor, as well as, etiologic factors such as the type of mutation and distinct types of injuries affecting different regions ${ }^{[20]}$.

Increased COX-2 expression in high grade OSCC noted in this study may be due to the fact that the malignant epithelial cells secrete factors like IL 10, MMP 2, MMP 9, MMP 13, ROS,VEGF, CXCL1, CXCL8, PDGF, IL-8, FGF-2, TGF- $\beta$, TNF- $\alpha$, IL-1 and granulocyte - macrophage colony stimulating factor (GMCSF) ${ }^{[29]}$.These factors induce inflammation with $\mathrm{COX}-2$ production in turn. Moreover, constitutive activation of NF- $x \mathrm{~B}$ reported in OSCC, further enhances the expression of pro-inflammatory cytokines, such as interleukin (IL)-1 $\alpha$, IL-6 and IL-8 with subsequent increase in COX-2 expression ${ }^{[35]}$.

Another reason may be the fact that, as the severity or grade of the cancerous condition increases, there is alteration in the levels of $\mathrm{p} 53$, a tumor suppressor gene which increases COX-2 expression ${ }^{[29]}$.

It may also be argued that conversely COX-2 is a contributing factor in the increased tumor grade and metastasis seen in OSCC. This may take place through direct or indirect mechanisms ${ }^{[36]}$, directly 
through promoting mitotic activity and conversion of pro-carcinogens to carcinogens and indirectly through increasing the blood vessels in the TME ${ }^{[32]}$.

Moreover, Li M. et al. (2015) ${ }^{[7]}$ recently documented that TAMs in the TME can express COX2 which results in increased proliferation, survival and increased angiogenesis and hence tumor grade. This is due to the over-expression of PGE2 with subsequent increase in $\mathrm{Bcl}-2$ and c-AMP ${ }^{[37]}$ which activate kinases such as PKA or PI3K and inhibition of GSK3 leading to activation of the $\beta$-catenin, eventually increasing tumor grade ${ }^{[35]}$.

Whether poorly differentiated OSCC increases COX-2 expression or COX-2 increases OSCC grade are both possible scenarios, but the fact remains that in this study COX-2 expression was elevated with increased OSCC grade. This may allow the prediction of the prognosis or even present a possible therapeutic option where COX2 inhibitors may be used as an adjuvant for cancer therapy, as COX-2 inhibitors are readily available and have fewer side effects than conventional cancer therapy.

In this study, immunohistochemical staining for CD163 in well and moderately differentiated OSCC was mainly confined to the connective tissue in close proximity to the malignant epithelial cells. However, the poorly differentiated cases revealed more diffuse immunopositivity of CD163 positive cells than in well and moderately differentiated ones. The expression was mostly noted in the stromal cells. Although CD163 is a specific marker for M2 macrophages, a small number of immunopositive cells were noted in the tumor islets in this study.

This phenomenon was also documented by Maniecki M. et al.(2012) ${ }^{[38]}$ who stated that there are several possible mechanisms that could explain the means by which distinct cancer cells undergo a phenotypic shift harboring molecular pathways that are normally only present in macrophages. It may result from a horizontal transfer of mRNA by microvesicles shed from macrophages or from heterotypic cell fusion between tumor cells and CD163 expressing TAMs.

In this study, it was observed that the reaction was granular in nature. These results were consistent with those of Barbosa N. et al (2015) ${ }^{[39]}$ and Cheng Z. et al. (2017) $)^{[40]}$ who stated that CD163 mRNA is upregulated in the cytoplasm of stromal cells in TME of OSCC, which may explain the granular reaction reported in this study. Also the nuclear reaction may be due to the increased transcription for the CD163 protein during the polarization macrophages to the M2 cells.

Similar to the COX-2 expression in this study, CD163 expression was significantly higher in the poorly differentiated OSCC compared to its expression in the well and moderately differentiated ones. There was an insignificant difference in CD163 expression between the well and moderately differentiated OSCC in this study. These results were similar to those of GochevaV. et al. (2010) ${ }^{[41]}$, Liu C. et al.( 2013) ${ }^{[42]}$ and Fan Q. et al. $(2014)^{[43]}$, who also described an up-regulation in CD163 expression in higher grade malignancies. In these studies, CD163 was used as a marker to assess M2 macrophages distribution in the TME.

The increase in the CD163 positive cells in higher grade tumors and the distribution of the CD163 positive cells in close proximity to the malignant epithelial cells noted in this study, may be explained by the fact that tumor cells actively produce microparticles, CCL2/3/4, CSF1, IL-4 and IL-10 which attract the CD163 positive cells. Moreover, tissue stress (hypoxia, tumor-derived HMGB-1, ECM components and VEGF) results in the increase of CD163 positive cells (M2) polarization $^{[14,44-46]}$.

At the same time, the production of TGF- $\beta$ by CD163 positive cells (M2) contributes to the general suppression of anti-tumor immunity. TGF- $\beta$ is a key regulator of the signaling pathways that initiate and maintain Foxp3 expression and inhibition of Th1 
immune cells and CTLs which play key roles in proliferation, apoptosis, metastasis, cell metabolism, aging and cancer biology [47, 48]. TGF- $\beta$ induces IL-6 and proteases like cathepsin B, MMPs which cleave the ECM releasing sequestrated VEGF ${ }^{[49}, \underline{50]}$ Altogether, these factors act to increase the grade of malignancy which explain the positive correlation that exists between CD163 positive cells and tumor grade observed in this study.

Moreover, Liu C. et al. (2013) ${ }^{[42]}$ showed that TAMs promoted EMT in pancreatic cancer cells, partially through the TLR4/IL-10 signaling pathway.

Reports also stated that CD163 positive cells (M2) promote CSC-like properties that have the potential to initiate tumor formation by undergoing self-renewal, differentiation $[\underline{51}, \underline{52}]$ via TGF- $\beta 1$ induced EMT in cancer ${ }^{[43]}$.

Moreover CD163 positive cells result in constitutive $\mathrm{NF}^{-}{ }_{\mathrm{K}}$ Bactivation which induces several cellular modifications associated with tumorigenesis and more aggressive phenotypes, including selfsufficiency in growth signals, insensitivity to growth inhibition, resistance to apoptotic signals, angiogenesis, migration and tissue invasion ${ }^{[\underline{53}]}$.

The results of this study revealed as well that a positive correlation exists between expression of the COX-2 and CD163 in different grades of OSCC. This may be due to the fact that COX-2 over-expression in TME particularly macrophages, enhances tumor progression and is essential for induction and maintenance of $\mathrm{M} 2^{[14]}$.

Moreover, the elevated PGE2 in the TME promotes COX-2 and CD163 positive (M2) polarization, in part via induction of the c-AMP ${ }^{[3}$, ${ }^{5,54]}$. On the other hand, these CD163 positive (M2) are in turn are the major source of COX-2 which induces PGE2 production from arachidonic acid (AA) during inflammation ${ }^{[55]}$ and so forth. From the above, it can be deducted that COX-2 causes the upregulation of CD163 expression and vice versa.
Both these molecules appear to act synergistically in increasing the grade of malignancy through several cytokines like the TGF- $\beta$ that both COX-2 and CD163 are thought to increase the number of CSCs in the tumors. It has been reported that the localization of COX-2 expression from the nucleus to the cytoplasm was associated with increased Oct4 and increased stem like properties of cells. CD163 also increases CSCs as discussed earlier and hence both these molecules contribute to the increased tumor grade. Finally, the increase in both $\mathrm{COX}-2$ and CD163 may also result from factors secreted from the malignant epithelial cells themselves ${ }^{[56]}$.

Thus, it is reasonable to think that in the future COX-2 and TAM modulating therapies might be at the disposal of clinicians and patients ${ }^{[57]}$. Nevertheless, the fact that there is a wide variation in pathways and factors that lead to or affect oral carcinogenesis remains.

\section{CONCLUSIONS}

From the present study we could conclude that the inflammatory signals presented by COX-2 immunoexpression and TAM (CD163 ${ }^{+}$cells) were elevated with increased tumor grade and thus, may have a role in the progression of OSCC.

\section{RECOMMENDATIONS}

Further studies are required to determine which of these mechanisms are more important in the development and progression of OSCC.

\section{REFERENCES}

1. Nakagaki, T., et al., Profiling cancer-related gene mutations in oral squamous cell carcinoma from Japanese patients by targeted amplicon sequencing. Oncotarget, 2017. 8(35): p. 59113.

2. Balkwill, F. and A. Mantovani, Inflammation and cancer: back to Virchow? The lancet, 2001. 357(9255): p. 539-545.

3. Chen, E.P. and E.M. Smyth, COX-2 and PGE2-dependent immunomodulation in breast cancer. Prostaglandins \& other lipid mediators, 2011. 96(1-4): p. 14-20. 
4. Lu Gan,Z.Q., et al., Cyclooxygenase-2 in tumor-associated macrophages promotes metastatic potential of breast cancer cells through Akt pathway. International journal of biological sciences, 2016. 12(12): p. 1533.

5. Hangai, S., et al., PGE2 induced in and released by dying cells functions as an inhibitory DAMP. Proceedings of the National Academy of Sciences, 2016. 113(14): p. 38443849 .

6. Kis, B., et al., Putative cyclooxygenase-3 expression in rat brain cells. Journal of Cerebral Blood Flow \& Metabolism, 2003. 23(11): p. 1287-1292.

7. Li, H., et al., Cyclooxygenase-2 in tumor-associated macrophages promotes breast cancer cell survival by triggering a positive-feedback loop between macrophages and cancer cells. Oncotarget, 2015. 6(30): p. 29637.

8. Mori, K., et al., Infiltration of $\mathrm{m} 2$ tumor-associated macrophages in oral squamous cell carcinoma correlates with tumor malignancy. Cancers, 2011. 3(4): p. 37263739 .

9. Pettersen, J.S., et al., Tumor-associated macrophages in the cutaneous SCC microenvironment are heterogeneously activated. Journal of Investigative Dermatology, 2011. 131(6): p. 1322-1330.

10. Guo, Q., et al., New mechanisms of tumor-associated macrophages on promoting tumor progression: recent research advances and potential targets for tumor immunotherapy. Journal of immunology research, 2016. 2016. Article ID 9720912, 12 pages. http://dx.doi. org/10.1155/2016/9720912

11. Zhi, Y., et al., Clinical significance of sCD163 and its possible role in asthma. Molecular Medicine Reports, 2017. 15(5): p. 2931-2939.

12. Solinas, G., et al., Tumor-conditioned macrophages secrete migration-stimulating factor: a new marker for M2polarization, influencing tumor cell motility. The Journal of Immunology, 2010. 185(1): p. 642-652.

13. Matsumoto-Okazaki, Y., J. Yamane, and K. Kajiya, Realtime imaging of interaction between macrophages and lymphatic vessels in an in vitro model to study inflammatory resolution. Journal of dermatological science, 2015. 77(1): p. 76-79.

14. Yang, L. and Y. Zhang, Tumor-associated macrophages: from basic research to clinical application. Journal of hematology \& oncology, 2017. 10(1): p. 58.
15. Rivera, C. and B. Venegas, Histological and molecular aspects of oral squamous cell carcinoma. Oncology letters, 2014. 8(1): p. 7-11.

16. Rakoff-Nahoum, S., Cancer issue: why cancer and inflammation? The Yale journal of biology and medicine, 2006. 79(3-4): p. 123.

17. Schetter, A.J., N.H. Heegaard, and C.C. Harris, Inflammation and cancer: interweaving microRNA, free radical, cytokine and p53 pathways. Carcinogenesis, 2009. 31(1): p. 37-49.

18. Reuter, S., et al., Oxidative stress, inflammation, and cancer: how are they linked? Free Radical Biology and Medicine, 2010. 49(11): p. 1603-1616.

19. Compare, D., O. Nardone, and G. Nardone, Non-steroidal anti-inflammatory drugs in the carcinogenesis of the gastrointestinal tract. Pharmaceuticals, 2010. 3(8): p. 2495 2516.

20. Seyedmajidi, M., et al., Cyclo-oxygenase-2 expression in oral squamous cell carcinoma. Journal of cancer research and therapeutics, 2014. 10(4): p. 1024.

21. Itoh, S., et al., Immunohistochemical study on overexpression of cyclooxygenase- 2 in squamous cell carcinoma of the oral cavity: its importance as a prognostic predictor. Oral oncology, 2003. 39(8): p. 829-835.

22. Pandey, M., et al., Overexpression of COX-2 gene in oral cancer is independent of stage of disease and degree of differentiation. International journal of oral and maxillofacial surgery, 2008. 37(4): p. 379-383.

23. Goulart Filho, J.A.V., et al., Immunoexpression of cyclooxygenase- 2 and p53 in oral squamous cell carcinoma. American journal of otolaryngology, 2009. 30(2): p. 89-94.

24. Urade, M., Cyclooxygenase (COX)-2 as a potent molecular target for prevention and therapy of oral cancer. Japanese Dental Science Review, 2008. 44(1): p. 57-65.

25. Zaynagetdinov, R., et al., A critical role for macrophages in promotion of urethane-induced lung carcinogenesis. The Journal of Immunology, 2011. 187(11): p. 5703-5711.

26. Zhang, J., et al., Regulation of epithelial-mesenchymal transition by tumor-associated macrophages in cancer. American journal of translational research, 2015. 7(10): p. 1699.

27. Shabo, I., et al., Breast cancer expression of CD163, a macrophage scavenger receptor, is related to early dis- 
tant recurrence and reduced patient survival. International Journal of Cancer, 2008. 123(4): p. 780-786.

28. Nagatsuka, H., et al., Heparanase and cyclooxygenase-2 gene and protein expressions during progression of oral epithelial dysplasia to carcinoma. Annals of diagnostic pathology, 2012. 16(5): p. 354-361.

29. Chiu, H.-F., et al., Cyclooxygenase-2 expression in oral precancerous and cancerous conditions and its inhibition by caffeic acid phenyl ester-enriched propolis in human oral epidermal carcinoma KB cells. Archives of Biological Sciences, 2017.69(1): p. 83-91.

30. Shirahama, T. and C. Sakakura, Overexpression of cyclooxygenase- 2 in squamous cell carcinoma of the urinary bladder. Clinical Cancer Research, 2001. 7(3): p. 558-561.

31. Parfenova, H., et al., Dynamics of nuclear localization sites for COX-2 in vascular endothelial cells. American Journal of Physiology-Cell Physiology, 2001. 281(1): p. C166-C178.

32. Masferrer, J.L., et al., Antiangiogenic and antitumor activities of cyclooxygenase-2 inhibitors. Cancer research, 2000. 60(5): p. 1306-1311.

33. Renkonen, J., H. Wolff, and T. Paavonen, Expression of cyclo-oxygenase-2 in human tongue carcinoma and its precursor lesions. Virchows Archiv, 2002. 440(6): p. 594597.

34. Shibata, M., et al., Cyclo-oxygenase-1 and-2 expression in human oral mucosa, dysplasias and squamous cell carcinomas and their pathological significance. Oral oncology, 2005. 41(3): p. 304-312.

35. Sobolewski, C., et al., The role of cyclooxygenase- 2 in cell proliferation and cell death in human malignancies. International journal of cell biology, 2010. 2010.

36. Jimi, E., et al., NF- $\varkappa$ B acts as a multifunctional modulator in bone invasion by oral squamous cell carcinoma. Oral Science International, 2016. 13(1): p. 1-6.

37. Sheng, H., et al., Modulation of apoptosis and Bcl-2 expression by prostaglandin E2 in human colon cancer cells. Cancer research, 1998. 58(2): p. 362-366.

38. Maniecki, M.B., et al., Tumor-promoting macrophages induce the expression of the macrophage-specific receptor CD163 in malignant cells. International journal of cancer, 2012. 131(10): p. 2320-2331.
39. Barbosa, N.G., et al., Immunohistochemical study of macrophages subpopulations associated with squamous cell carcinoma of the tongue, with and without metastasis. Jornal Brasileiro de Patologia e Medicina Laboratorial, 2015. 51(6): p. 415-421.

40. Cheng, Z., et al., CD163 as a novel target gene of STAT3 is a potential therapeutic target for gastric cancer. Oncotarget, 2017. 8(50): p. 87244.

41. Gocheva, V., et al., IL-4 induces cathepsin protease activity in tumor-associated macrophages to promote cancer growth and invasion. Genes \& development, 2010. 24(3): p. 241-255.

42. Liu, C.-Y., et al., M2-polarized tumor-associated macrophages promoted epithelial-mesenchymal transition in pancreatic cancer cells, partially through TLR4/IL-10 signaling pathway. Laboratory investigation, 2013. 93(7): p. 844.

43. Fan, Q.-M., et al., Tumor-associated macrophages promote cancer stem cell-like properties via transforming growth factor-beta1-induced epithelial-mesenchymal transition in hepatocellular carcinoma. Cancer letters, 2014. 352(2): p. 160-168.

44. Lewis, C.E. and J.W. Pollard, Distinct role of macrophages in different tumor microenvironments. Cancer research, 2006. 66(2): p. 605-612.

45. Mantovani,A., et al., Cancer-related inflammation. Nature, 2008. 454(7203): p. 436.

46. Murdoch, C., et al., Expression of Tie-2 by human monocytes and their responses to angiopoietin-2. The Journal of Immunology, 2007. 178(11): p. 7405-7411.

47. Zamarron, B.F. and W. Chen, Dual roles of immune cells and their factors in cancer development and progression. Int J Biol Sci, 2011. 7(5): p. 651-658.

48. Liu, S.-Y., et al., Clinicopathologic significance of tumor cell-lined vessel and microenvironment in oral squamous cell carcinoma. Oral oncology, 2008. 44(3): p. 277-285.

49. Deng, Y.-R., et al., Sorafenib inhibits macrophage-mediated epithelial-mesenchymal transition in hepatocellular carcinoma. Oncotarget, 2016. 7(25): p. 38292.

50. Qian, B.-Z. and J.W. Pollard, Macrophage diversity enhances tumor progression and metastasis. Cell, 2010. 141(1): p. 39-51. 
51. Aras, S. and M.R. Zaidi, TAMeless traitors: macrophages in cancer progression and metastasis. British journal of cancer, 2017. 117(11): p. 1583.

52. Wu, A., et al., Glioma cancer stem cells induce immunosuppressive macrophages/microglia. Neuro-oncology, 2010. 12(11): p. 1113-1125.

53. Xia, Y., S. Shen, and I.M. Verma, NF- $x$ B, an active player in human cancers. Cancer immunology research, 2014. 2(9): p. 823-830.

54. Luan, B., et al., CREB pathway links PGE2 signaling with macrophage polarization. Proceedings of the National Academy of Sciences, 2015. 112(51): p. 15642-15647.
55. Williams, J.A. and E. Shacter, Regulation of Macrophage Cytokine Production by Prostaglandin E2 Distinct roles of cyclooxygenase-1 and-2. Journal of Biological Chemistry, 1997. 272(41): p. 25693-25699.

56. Thanan, R., et al., Nuclear localization of COX-2 in relation to the expression of stemness markers in urinary bladder cancer. Mediators of inflammation, 2012. 2012. Article ID 165879, 8 pages. http://dx.doi.org/10.1155/2012/165879

57. Sousa, S. and J. Määttä, The role of tumour-associated macrophages in bone metastasis. Journal of bone oncology, 2016. 5(3): p. 135-138. 